\title{
Elastic Properties of GaN Nanowires: Revealing the influence of planar defects on Young's modulus at nanoscale
}

\author{
Sheng Dai ${ }^{1,2}$, Jiong Zhao ${ }^{1}$, Xiaoguang Wang ${ }^{3}$, Zhiwei Shan ${ }^{3}$, Jing Zhu ${ }^{1}$ \\ 1. Beijing National Center for Electron Microscopy, School of Materials Science and Engineering, \\ Tsinghua University, Beijing, 100084, China \\ 2. Department of Materials Science and Engineering, University of Michigan, Ann Arbor, Michigan \\ 48109, USA. \\ 3. Center for Advancing Materials Performance from the Nanoscale (CAMP-Nano) \& Hysitron Applied \\ Research Center in China (HARCC), Xi'an Jiaotong University, Xi'an, 710049, China.
}

Gallium nitride $(\mathrm{GaN})$ nanowire, a technologically important semiconductor has ignited the research attention in recent years. This materials has also been investigated extensively and proved to process enhanced physical properties and nanodevice applications. Besides its well-known optoelectronic and photonic properties, the study on the mechanical properties of $\mathrm{GaN}$ nanowires is essential and imperative for its piezoelectric applications and the designing for GaN-nanowire-based devices during processing and working.

In our experiments, $\mathrm{GaN}$ nanowires with different structures were synthesized via chemical vapor deposition method. Both single crystalline (SC) GaN nanowires and a new structure, obtuse-angle twin (OT) GaN nanowires were obtained. High resolution electron microscopy was utilized to characterize the structures and the planar defects such as twin boundaries and stacking faults. The results (Figure 1) showed that (001) stacking faults have different relative orientations and volume fractions in SC and OT GaN nanowires, respectively. This is an ideal system for the investigation to explore the influence of planar defects on Young's modulus at nanoscale.

In-situ electric-field-induced resonance method was utilized to reveal that SC GaN nanowires, along [120] direction, had the similar Young's modulus as the bulk value at the diameter ranging 92-110 $\mathrm{nm}$. Meantime, the elastic behavior of the obtuse-angle twin (OT) GaN nanowires was disclosed both by insitu SEM resonance technique and in-situ TEM tensile test for the first time (Figure 2). Our results showed that the average Young's modulus of these OT nanowires was greatly decreased to about 66 $\mathrm{GPa}$ and indicated no size dependence at the diameter ranging 98-171 nm. A quantitative model based on the rules of mixture is proposed and the calculation results are in great accordance with our experimental data both in two kinds of GaN NWs.

Our work demonstrated that the elastic modulus of one dimensional nanomaterials is dependent on the relative orientations and the volume fractions of planar defects. The Voigt model and Reuss model in classical mechanics still remain effective in our tested GaN nanowires and may be extended to some other one-dimensional namomaterials. Moreover, the SFs' effect on the elastic properties also indicates that one may construct new type of functional devices through planar defects engineering. 


\section{References:}

[1] S Dai, J Zhao, M He et al. Nano Letters 15 (2015), p.8.

[2] S Dai, J Zhao, M He et al. J. Phys. Chem. C 117 (2013), p.12895.

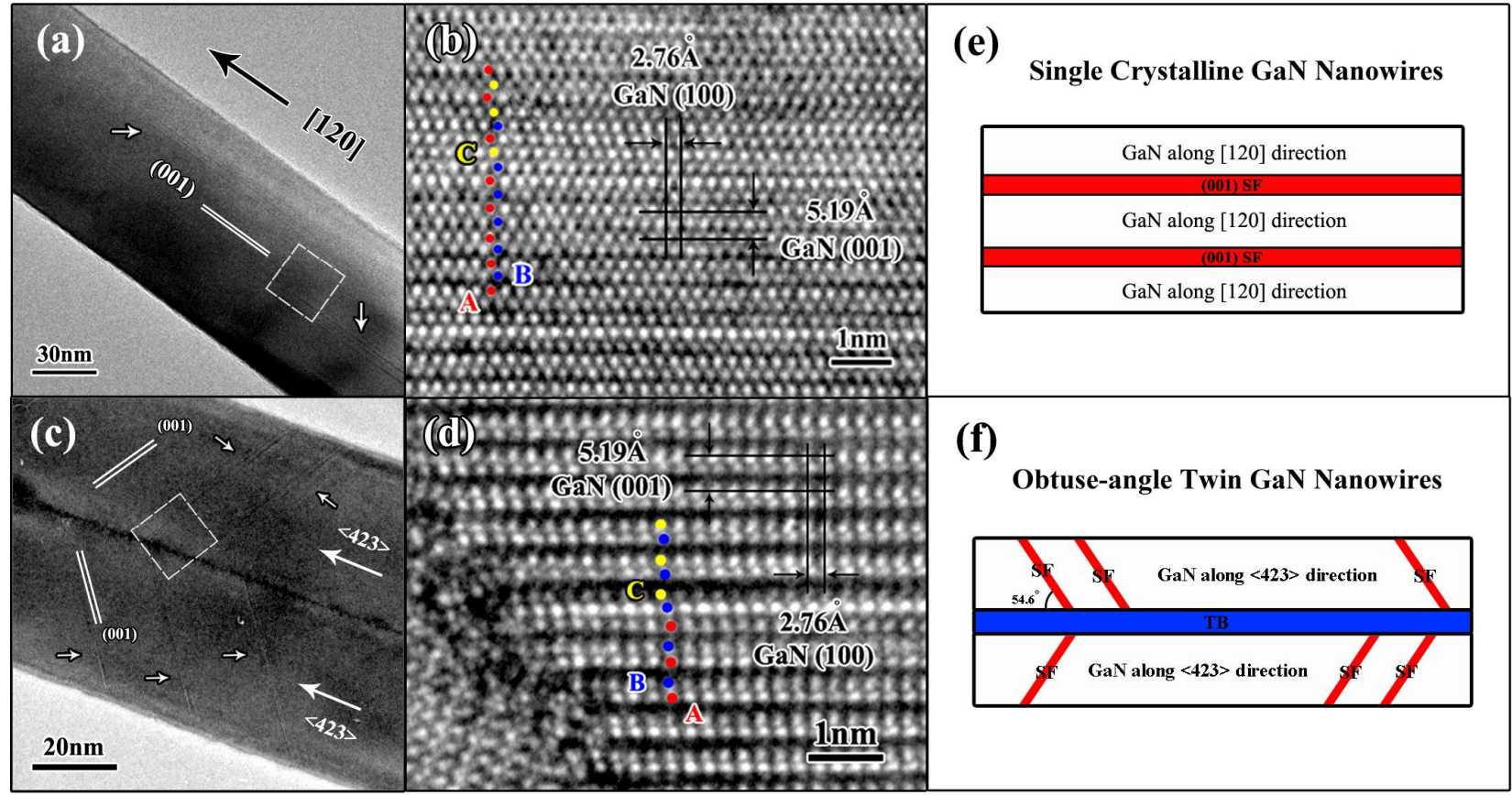

Figure 1. HRTEM characterizations of GaN nanowires showing the planar defects and structure model. $(a, b, e)$ are the results of single crystalline GaN nanowires. (c, d, f) are the results of obtuse-angle twin GaN nanowires.
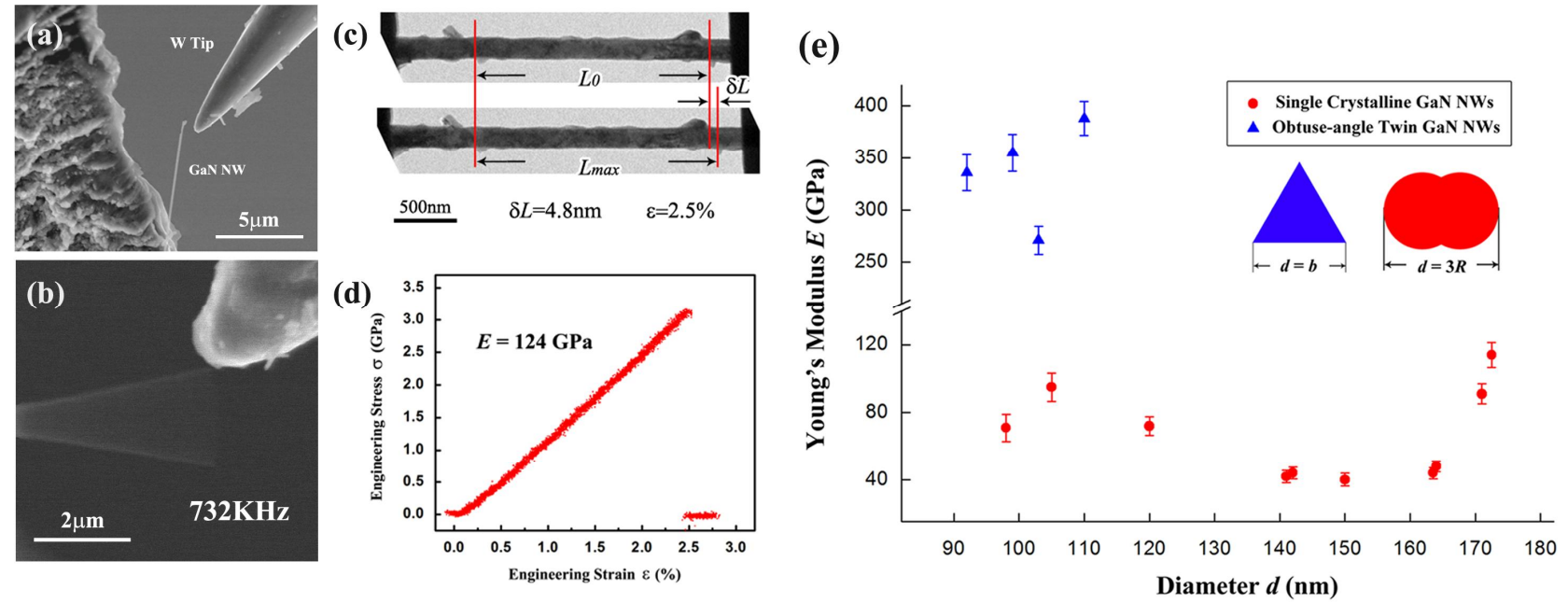

Figure 2. In-situ experiments to measure the Young's modulus of as-synthesized GaN naonowires. (a-b) In-situ SEM electric-field-induced resonance. (c-d) In-situ TEM tensile tests. (e) Young's Modulus $E$ with Diameter $d$ of GaN nanowires from resonance experiments. 